\title{
632 TORIPALIMAB COMBINED WITH PLATINUM-BASED CHEMOTHERAPY AS A SECOND-LINE TREATMENT FOR SMALL CELL LUNG CANCER (SCLC) WITH BRAIN METASTASES: A CASE REPORT
}

Jieqiong Fan*, Yao Zhang, Xiaofei Mo, Jing Guo. Chongqing University Three Gorges Hospital, Chongqing, China

Background Immunotherapy targeting PD-L1 together with platinum-based chemotherapy has demonstrated clinical activity in extensive-stage small cell lung cancer (ES-SCLC). However, brain metastasis seems to be an adverse prognostic factor. Here we report a case of a pretreated ES-SCLC patient with brain metastases who obtained marked clinical benefit from a combination of the anti-PD-1 antibody (toripalimab) together with platinum-based chemotherapy.

Methods A 56-year-old male smoker first presented at our hospital on March 17, 2018, with a dull pain in the right chest wall, without obvious cause. The patient underwent a biopsy of the right lung, revealing small cell carcinoma. Between April 20, 2018 and June 27, 2018, the patient received four cycles of platinum-based chemotherapy (irinotecan with carboplatin) and achieved a partial response (PR) (RECIST 1.1). However, at the end of 2019, his condition began to deteriorate. He had left upper abdominal pain without obvious cause, followed by dizziness, headache, unsteady walking, dull pain in the middle and upper abdomen, accompanied by acid regurgitation and belching. CT and MRI showed secondary malignant tumors in the liver, intracranially and in the pancreas with lymph node metastasis. Because the patient refused radiotherapy, from June 2020 he was treated with etoposide (160 mg, every 3 weeks on day 1-3) plus cisplatin (40 mg, every 3 weeks on day $1-3$ ) combined with toripalimab (240 mg, every 3 weeks on day 1$)$.

Results Following two cycles of treatment, MRI showed significant shrinkage of the patient's intracranial tumor. After the next sequential 4 cycles of therapy, MRI showed that the intracranial tumor had almost disappeared, CT images showed similar shrinkage of the liver and pancreas tumors. The overall efficacy assessment was PR. The patient tolerated the treatment with no side effects and is experiencing a durable clinical benefit. He now maintains immunotherapy and leads a normal life with no progression at the latest follow up on June 2021.

Conclusions Immunotherapy targeting PD-L1 added to platinum-based chemotherapy has been shown to prolong overall survival in ES-SCLC patients. Even in pretreated patients with brain metastases, PD-1 inhibitors plus platinum-based chemotherapy may also play a potential role. This is worthy of further investigation in formal clinical trials.

http://dx.doi.org/10.1136/jitc-2021-SITC2021.632 